\title{
First genomic insights into members of a candidate bacterial phylum responsible for wastewater bulking
}

Yuji Sekiguchi, Akiko Ohashi, Donovan H Parks, Toshihiro Yamauchi, Gene W Tyson, Philip Hugenholtz

Filamentous cells belonging to the candidate bacterial phylum KSB3 were previously identified as the causative agent of fatal filament overgrowth (bulking) in a high-rate industrial anaerobic wastewater treatment bioreactor. Here, we obtained near complete genomes from two KSB3 populations in the bioreactor, including the dominant bulking filament, using differential coverage binning of metagenomic data. Fluorescence in situ hybridization with 16S rRNA-targeted probes specific for the two populations confirmed that both are filamentous organisms. Genome-based metabolic reconstruction and microscopic observation of the KSB3 filaments in the presence of sugar gradients indicate that both filament types are Gram-negative, strictly anaerobic fermenters capable of nonflagellar based gliding motility, and have a strikingly large number of sensory and response regulator genes. We propose that the KSB3 filaments are highly sensitive to their surroundings and that cellular processes, including those causing bulking, are controlled by external stimuli. The filaments are shown to chemotactically respond to glucose gradients, and by keeping this sugar low in the industrial bioreactor, no further bulking has occurred to date. The obtained genomes lay the foundation for a more detailed understanding of environmental cues used by KSB3 filaments, which may lead to more robust treatment options to prevent bulking. 
1 First genomic insights into members of a candidate bacterial phylum responsible for

2 wastewater bulking

3 Yuji Sekiguchi ${ }^{1}$, Akiko Ohashi ${ }^{1}$, Donovan H. Parks ${ }^{2}$, Toshihiro Yamauchi ${ }^{3}$,

4 Gene W. Tyson ${ }^{2,4}$, and Philip Hugenholtz ${ }^{2,5}$

$5{ }^{1}$ Biomedical Research Institute, National Institute of Advanced Industrial Science and

6 Technology (AIST), Tsukuba, Ibaraki, Japan

$7{ }^{2}$ Australian Centre for Ecogenomics, School of Chemistry and Molecular Biosciences, The 8 University of Queensland, St. Lucia, Queensland, Australia.

$9 \quad{ }^{3}$ Administrative Management Department, Kubota Kasui Corporation, Minato-ku, Tokyo, Japan.

$10{ }^{4}$ Advanced Water Management Centre, The University of Queensland, St. Lucia, Queensland, 11 Australia.

$12{ }^{5}$ Institute for Molecular Bioscience, The University of Queensland, St. Lucia, Queensland, 13 Australia.

14 Correspondence Author: Yuji Sekiguchi

15 Address: Biomedical Research Institute, National Institute of Advanced 16

17

18 Phone:

19 Email: Industrial Science and Technology (AIST), AIST Tsukuba Central 6, Ibaraki 305-8566, Japan.

(+81) 298617866

y.sekiguchi@aist.go.jp

20 Correspondence Author: Philip Hugenholtz

21 Address:

22

23 Phone:

24 Email:

Australian Center for Ecogenomics, Building 76, The University of Queensland, St Lucia, QLD 4072, Australia.

(+617) 33653822

p.hugenholtz@uq.edu.au 


\section{Abstract:}

26 Filamentous cells belonging to the candidate bacterial phylum KSB3 were previously identified

27 as the causative agent of fatal filament overgrowth (bulking) in a high-rate industrial anaerobic

28 wastewater treatment bioreactor. Here, we obtained near complete genomes from two KSB3 29 populations in the bioreactor, including the dominant bulking filament, using differential 30 coverage binning of metagenomic data. Fluorescence in situ hybridization with 16S rRNA31 targeted probes specific for the two populations confirmed that both are filamentous organisms.

32 Genome-based metabolic reconstruction indicate that both filament types are Gram-negative, 33 strictly anaerobic fermenters that have a strikingly large number of sensory and response 34 regulator genes. Microscopic observations of the KSB3 filaments show that they chemotactically 35 respond to maltose and glucose gradients by non-flagellar based gliding motility. We propose that 36 the KSB3 filaments are highly sensitive to their surroundings and that cellular processes, 37 including those causing bulking, are controlled by external stimuli. The obtained genomes lay the 38 foundation for a more detailed understanding of environmental cues used by KSB3 filaments, 39 which may lead to more robust treatment options to prevent bulking.

\section{Introduction}

41 Anaerobic digestion is a major type of biological treatment extensively used around the world 42 (Ahring, 2003a) that is not only cost effective for treating organic waste and wastewater, but also 43 can frequently produce energy in the form of methane (biogas) (Angelidaki et al., 2011). Over the 44 last thirty years, a set of high rate anaerobic digestion reactor configurations have been 45 developed, of which the upflow anaerobic sludge blanket (UASB) technology is the most 46 successful and commercialized configuration (Kleerebezem \& Macarie, 2003; van Lier, 2008).

47 Despite the success of this technology, serious performance issues have emerged such as the 
48 sudden washout of granular sludge biomass due to overgrowth of filamentous bacteria (bulking),

49 which can lead to complete loss of performance.

50 Bulking of anaerobic digestion systems can be caused by a variety of filamentous

51 microorganisms (Hulshoff Pol et al., 2004; Li et al., 2008; Yamada \& Sekiguchi, 2009) and a

52 phylogenetically novel filament was previously reported to be the cause of bulking in an

53 industrial UASB reactor treating sugar manufacturing wastewater (Yamada et al., 2007; 2011).

54 Small subunit ribosomal RNA (16S rRNA) gene-based analyses of the bulking sludge (Yamada

55 et al., 2007) revealed that the dominant filament type belongs to candidate bacterial phylum

56 KSB3, originally proposed by Tanner et al. (2000) based on an environmental 16S rRNA gene

57 clone sequence obtained from a sulfur-rich marine sediment (Tanner et al., 2000). Fluorescence

58 in situ hybridization (FISH) with KSB3-specific 16S rRNA-directed probes revealed that the

59 KSB3 filaments are localized at the outer layer of healthy granules (Yamada et al., 2007) which

60 become substantially thicker during bulking. The study of filamentous KSB3 bacteria will

61 undoubtedly contribute to our understanding of and ability to prevent bulking in anaerobic

62 wastewater treatment systems, but has been hampered by an inability to obtain a pure culture

63 despite repeated and long term isolation efforts (Yamada et al., 2011). However, culture-

64 independent molecular and imaging methods are beginning to provide clues regarding the

65 ecophysiology of these organisms. This includes their ability to uptake simple carbohydrates,

66 particularly maltose and glucose, under anaerobic conditions and from these observations it was

67 proposed that high carbohydrate loading in the UASB reactor may trigger proliferation of KSB

68 filament populations (Yamada et al., 2011).

69 Here, we obtained near complete genomes from in situ populations of the dominant bulking

70 KSB3 filament type and a second moderately related low abundance KSB3 filament via 
71 differential coverage binning (Albertsen et al., 2013) using metagenomic data previously reported

72 from a full-scale UASB reactor (Soo et al., 2014). Differential coverage binning groups together

73 anonymous metagenomic fragments (contigs) belonging to the same population based on the

74 similarity of their sequencing coverage across multiple related metagenomes (Albertsen et al.,

75 2013). These genomes represent the first genomic information for candidate phylum KSB3 and

76 provide insights into the metabolism of KSB3 filaments and their ability to cause bulking.

\section{Methods}

78 Samples. Methanogenic sludge samples reported in a previous study (Soo et al., 2014) were used 79 in the present study for shotgun sequencing and fluorescence in situ hybridization (FISH). 80 Briefly, two sludge samples (A1 and A2) were taken from the system at different sampling dates 81 (A1, 25 ${ }^{\text {th }}$ December, 2012; A2, 16 ${ }^{\text {th }}$ September, 2010), and sample A1 was further separated into 82 two parts (flocculant sludge [F1] and granular sludges [G1]) by gravimetric settlement (Soo et al., 83 2014) (Supplementary Table S1). Each sample had been divided into two parts, one used for 84 obtaining DNA via bead-beating and phenol chloroform extraction and the other fixed in $4 \%$ 85 paraformaldehyde for FISH (Soo et al., 2014).

87 Fluorescence in situ hybridization. KSB3-specific FISH probes were designed in ARB v5.5 88 (Ludwig et al., 2004) using 16S rRNA genes identified in the KSB3 genomes and KSB3 16S 89 rRNA gene sequences available in the current Greengenes database (May 2013 version) 90 (McDonald et al., 2012). In order to maximize the specificity and fluorescence intensity of the 91 probes, helper probes were also designed (Supplementary Table S5). FISH was performed as 92 described previously (Sekiguchi et al., 1999) using the probes listed in Supplementary Table S5 93 and all probes were hybridized overnight. Probes were labeled with either Alexa488 or Cy3 94 fluorophores and probes with different fluorophores were used together for dual-staining FISH, 
95 as shown in Fig. 1b. Images were captured using an epifluorescence microscope (Axioplan 2;

96 Carl Zeiss) equipped with a cooled charge-coupled device (CCD) camera (DP72; Olympus) and

97 subsequently processed using imaging software (DP2-BSW, version 2.2; Olympus). Super-

98 imposed images were generated using Adobe Photoshop (CS5.1; Adobe).

100 Metagenome sequencing. Previously sequenced paired-end and mate-pair metagenomes for 101 samples A1, A2, F1, and G1 (Soo et al., 2014) were supplemented with additional data generated 102 in this study using the same DNA. Paired-end Nextera libraries were prepared for each sample 103 according to the manufacturer's instructions, quantified using the QuantIT kit (Molecular Probes) 104 and sequenced ( 2 x 250 bp paired end) on an Illumina MiSeq using the Reagent Kit v2 (Illumina) 105 at the National Institute of Advanced Industrial Science and Technology, Japan (AIST; 106 Supplementary Table S1). The extra metagenome sequencing generated 7.3 Gb, $13.1 \mathrm{~Gb}, 1.9$ $107 \mathrm{Gbp}$, and $7.9 \mathrm{Gbp}$ for A1, A2, F1, and G1 samples, respectively (yielding a total of $56.2 \mathrm{~Gb}$ for 108 all samples combined). For scaffolding, two additional large-insert mate-pair libraries ( $\sim 3 \mathrm{kbp}$ 109 and $\sim 7.5 \mathrm{kbp}$ ) were constructed from sample A1 using the Mate Pair Library Preparation Kit v2 110 (Illumina) and sequenced on Illumina MiSeq system (MiSeq Reagent Kit v2) yielding $2.3 \mathrm{~Gb}$ and $1113.0 \mathrm{~Gb}$, respectively (for a total of $7.3 \mathrm{~Gb}$ from sample A1 when combined with mate-pair data; 112 Soo et al., 2014).

114 Community profiling. 16S rRNA gene amplicon sequencing of all UASB sludge samples using 115 the Illumina MiSeq system was previously reported (Soo et al., 2014). Community composition 116 was also examined by extracting all $16 \mathrm{~S}$ rRNA reads from the metagenome datasets using the 117 closed reference picking script in QIIME v1.6.0 (Caporaso et al., 2010b) with the Greengenes 118 database (de-replicated dataset at 97\%, March 2013 McDonald et al., 2012) as reference 119 (otu_picking_method, uclust_ref; similarity cutoff value, 0.95). All reads from paired end 
120 sequencing were quality filtered using a QIIME script (split_library_fastq.py) with the following

121 stringent parameters to ensure only high quality reads were included in the analysis: read

122 trimming with a Phred quality threshold of 17 (-q 17) and discarding reads shorter than $50 \%$ of

123 the input read length (-p 0.5), and then the quality filtered single-end reads were used for the

124 closed reference picking to generate OTU tables.

125

126 Assembly and binning. Metagenome assembly and population genome binning followed the

127 approach previously described (Albertsen et al., 2013). A graphical illustration of the workflow is

128 shown in Supplementary Figure S1. Briefly, paired-end metagenome reads in fastq format were

129 merged with SeqPrep (https://github.com/jstjohn/SeqPrep) using default settings and Illumina

130 sequencing adapters were removed. Unmerged reads were quality trimmed and filtered using

131 Nesoni v0.112 (https://github.com/ Victorian-Bioinformatics-Consortium/nesoni) with removing

132 low quality bases from reads with a Phred quality threshold of 17 , removing homopolymers

133 reads, and eliminating trimmed reads shorter than 30 bases (clip --quality 17 --homopolymers yes

134 --length 30). The merged and trimmed reads from the four metagenomes were co-assembled

135 using SPAdes v2.5.0 with the following parameters suited to a complex metagenomic assembly:

136 --only-assembler -k 67 --sc. Reads from respective samples were separately mapped to scaffolds

137 using BWA v0.7.4 (Li \& Durbin, 2010) with the BWA-MEM algorithm using default parameters.

138 Population genome binning using differential coverage (Albertsen et al., 2013) was performed

139 using GroopM v0.1 (Imelfort et al, 2014) with the initial core formation based on

140 contigs/scaffolds longer than 1,500 bp. Manual refinement of population genome bins, and

141 subsequent recruitment of contigs/scaffolds longer than $500 \mathrm{bp}$ was performed using the GroopM

142 tools.

143

144 Identification of conserved marker genes. All contigs/scaffolds in each genome bin were 
145 translated into six reading frames, and hmmsearch in HMMER3 (Eddy, 2011) was used to

146 identify 111 single copy marker genes conserved in most bacteria (Dupont et al., 2012), 83

147 phylogenetically-informative marker genes (Soo et al., 2014), and the 38 marker genes proposed

148 by PhyloSift (Darling et al., 2014). To determine the completeness and contamination of each

149 genome bin, the distribution and number of the 111 conserved single copy marker set was

150 determined using CheckM (Parks et al., 2014) with default settings.

151

152 Refinement of population genome bins. Scaffolding of metagenome contigs using the mate-pair

153 data was performed with SSPACE v2.0 (Boetzer et al., 2011). SSPACE was run with the

154 following two sets of parameters, lower stringency for minor population genomes with relatively

155 low coverage (e.g. UASB270): -k 2 (minimum number of links to compute scaffold) -a 0.7

156 (maximum link ratio between two best contig pairs) -x 0 (no extention of the contigs using paired

157 reads) -p 1 (making .dot file for visualization) and higher stringency for major population

158 genomes (e.g. UASB14): -k 4 -a 0.7 -x 0 -p 1. The resulting dot files were used for visualizing

159 contig connections using Cytoscape v.2.8.1 (Shannon et al., 2003). In addition, Cytoscape

160 attribute files were generated with coverage, length, and bin number (bin name) information for

161 each contig/scaffold. Based on the coverage information and number of connections between

162 contig/scaffolds, external contig/scaffolds are manually added to each bin. In addition,

163 contig/scaffolds with a small number of connections to other contig/scaffolds in their respective

164 bins were excluded. Refined sets of contig/scaffolds were then scaffolded with SSPACE with the

165 following low stringency parameters: -k 2 -a $0.7-\mathrm{x} 0$-p 1. For further refinement, shotgun mate-

166 pair reads were mapped to the newly generated scaffolds using CLC genomic workbench v6.0

167 (CLC Bio) using default parameters with the exception of a similarity fraction of 0.98 and

168 exported in SAM format. The assembly was visualized using Circos (Krzywinski et al., 2009)

169 and used for manual inspection of the assembly as previously described (Albertsen et al., 2013). 
170 Manual correction of misassembly and mis-scaffolding was performed using the microbial

171 genome finishing module in CLC genomic workbench v6.0 (CLC Bio).

\section{2}

173 Genome tree. Finished bacterial and archaeal genomes were downloaded from IMG (release 4.1)

174 (Markowitz et al., 2014), from which the 38 universally (Darling et al., 2014) or 83 single-copy

175 proteins broadly conserved in Bacteria were identified using HMM searches (Soo et al., 2014).

176 To evaluate the robustness of the protein trees (genome trees), four different outgroup taxon

177 configurations (two data sets for 38 marker genes, two data sets for 83 marker genes) were made

178 (Supplementary Table S3). Homologous proteins obtained from the KSB3 and reference

179 genomes in each taxon configuration were aligned using hmmalign in HMMER3, and

180 subsequently concatenated. A mask was generated for the concatenated alignment using Gblocks

181 (Talavera \& Castresana, 2007) with only conserved positions found in more than half of the

182 sequences considered. All tree topologies were tested for robustness using the maximum

183 likelihood methods from FastTree v2.1.7 (with default parameters, JTT model, CAT

184 approximation) (Price, Dehal \& Arkin, 2009) and RAxML v7.7.8 (JTT and Gamma models with

185 rapid 100 times bootstrapping) (Stamatakis, 2006). The PHYLIP SEQBOOT module

186 (Felsenstein, 1989) was used to generate 100 resampled alignments and FastTree was used to

187 analyze the resampled alignments (-n 100). A script (CompareToBootstrap.pl) included in the

188 FastTree package was used to compare the original tree to the resampled trees and generate

189 bootstrap values. Generated trees were imported into ARB (Ludwig et al., 2004), where they

190 were rooted, left-hand ladderized using the "beautify" tool and grouped into phylum-level clades.

191 A representative tree (tree no. 1; Supplementary Table S4) was exported from ARB and

192 visualized using iTOL (Letunic \& Bork, 2011).

193

194 16S rRNA gene phylogeny. KSB3 related 16S rRNA genes were manually curated using the 
195 Greengenes database (version May 2013; McDonald et al., 2012) in ARB (Ludwig et al., 2004).

196 16S rRNA genes from binned population genomes were aligned with PyNAST (Caporaso et al.,

197 2010a), imported into ARB, and the alignments were manually corrected using the ARB EDIT

198 tool. Sets of taxa (>1,300 nt) were selected in ARB and their alignments were exported applying

199 Lane mask filtering. One set of taxa included representatives across all recognized bacterial phyla

200 to determine the relative position of KSB3 in the bacterial domain (Supplementary Fig. S6). A

201 second set of taxa included all KSB3 sequences to determine the relative position of the two

202 UASB filament genomes within the KSB3 phylum (Fig. 1a). Neighbor joining trees were

203 calculated from the masked alignments with LogDet distance estimation using PAUP* 4.0

204 (Swofford, 2003) with 100 bootstrap resamplings. Maximum likelihood trees were calculated

205 based on the masked alignments using RAxML v7.7.8 (GTR and Gamma models + I) with rapid

206100 time bootstrapping. Maximum parsimony trees were calculated using PAUP* 4.0. A heuristic

207 search was used with a random stepwise addition sequence of 10 replicates and nearest-neighbor-

208 interchange swapping. Bootstrap analyses on the maximum parsimony trees were run with 100

209 times resampling for each best tree. Generated trees were re-imported into ARB for visualization.

210

211 Genome analysis. The two assembled KSB3 population genomes were initially annotated with

212 PROKKA v1.7 using default settings (Seemann, 2014), and manually curated by comparison to

213 UniRef90 (Suzek et al., 2007), IMG (Integrated Microbial Genomes, finished genomes, release

214 4.0) (Markowitz et al., 2014), COGs (Clusters of Orthologous Groups) (Tatusov et al., 2000),

215 PFAM (Punta et al., 2012), and KEGG (Kyoto Encyclopedia of Genes and Genomes) (Aoki-

216 Kinoshita \& Kanehisa, 2007) databases. Bi-directional best-blast matches were performed for

217 proteins with matches to UniRef90 and IMG using a bit score threshold of 300, and one-way

218 BLASTP matches with a bit score of 60 (Castelle et al., 2013). For COGs, RPS-BLAST against

219 COG PSSMs from the CDD database (Marchler-Bauer et al., 2013) was performed using an e- 
220 value cutoff of 0.01 , with the top hit retained for each protein domain. The amino acid sequences

221 were also searched for conserved motifs with PFAM (Punta et al., 2012) using HMMR3 (Eddy,

222 2011) and PfamScan with default settings (with family noise cutoff). Protein domain structure of

223 some gene products were additionally evaluated using InterProScan search (Quevillon et al.,

224 2005). For manual annotation of the KSB3 genomes, we ranked the resulting annotations as

225 follows: bi-directional best-blast matches with UniRef90 and IMG data; one-way matches with

226 UniRef90, IMG, and COGs; PFAM matches; hypothetical proteins (Castelle et al., 2013). For

227 comparison of gene sets with other genomes, we downloaded the full IMG database (release 4.1)

228 containing all genomes in IMG and their annotations (e.g. PFAMs and COGs). In addition, a list

229 of all finished bacterial genomes and associated metadata (e.g. taxonomic affiliation and genome

230 size) was obtained though IMG. Ribosomal RNA copy number was estimated by determining the

231 ratio of average genome coverage to 16S rRNA gene coverage for each KSB3 genome calculated

232 using BWA read mapping. CRISPR loci were identified using CRT v1.2 (Bland et al., 2007).

233 Presence/absence of some gene sets related to cell envelope structure (Albertsen et al., 2013),

234 complex bacterial lifestyle, and adaptability to fluctuating environmental conditions ('social IQ')

235 (Sirota-Madi et al., 2010) were evaluated based on IMG annotation for finished genomes and

236 annotated KSB3 genomes, and the resulting abundance matrix was visualized using $\mathrm{R}$ and

237 ggplot2. Orthologous proteins between the two KSB3 genomes were identified using pairwise bi-

238 directional best hit BLASTP searches. Glycoside hydrolases were identified using the CAZy

239 database (Lombard et al., 2014) (dbCAN HMMs v3.0, Yin et al., 2012) with HMMER3 (default

240 settings). Transmembrane proteins were predicted using TMHMM Server v. 2.0 (Moller, Croning

$241 \&$ Apweiler, 2002).

242 Gram-staining and gliding motility. Gram-staining for KSB3 filaments was performed based 243 on the method of Hucker (Doetsch, 1981). Gliding motility of KSB3 filaments was evaluated 
244 using fresh sludge samples examined under an epifluorescence microscope (Axioplan 2; Carl

245 Zeiss) equipped with an automatic thermo-control system (Thermo Plate, MATS-55SFG-FT;

246 Tokai Hit). Fresh sludge samples were placed on glass slides, and a cover glass carefully

247 positioned over the sample to minimize exposure to air. To maintain anaerobic conditions on the

248 slides, reducing agents $\left(\mathrm{Na}_{2} \mathrm{~S}\right.$ and/or L-cysteine) were added to the samples. The temperature of

249 the microscopic stage was maintained at $37^{\circ} \mathrm{C}$, and time-lapse images were recorded with a

250 cooled CCD camera (DP72; Olympus) equipped with the imaging software (DP2-BSW, version

251 2.2; Olympus). Glucose, maltose, ribose, mannose, galactose, arabinose, raffinose, sucrose,

252 xylose, fructose, lactate, ethanol, propionate, nitrate, nitrite (final concentration of 5-10 mM) and

253 yeast extract $(0.1 \%)$ were used as candidate stimuli to induce motility. Each potential stimulant

254 was mixed with the cells prior to the observation, or placed at the edge of the glass cover creating 255 a gradient as the stimulant diffused into the sample.

\section{Results}

\section{KSB3 populations in the UASB system}

258 Two UASB sludge samples taken two years apart (A1 and A2), and flocular (F1) and granular

259 (G1) fractions derived from sample A1, reported previously (Soo et al., 2014), were used in the

260 present study. The UASB system had a history of periodic bulking caused by KSB3 filaments

261 (Yamada et al., 2007; 2011). Inspection of 16S rRNA gene amplicon community profiles of these

262 samples (Soo et al., 2014) revealed two KSB3 16S rRNA phylotypes accounting for 4.9 and 3.7\%

263 of total sequencing reads from samples A1 and A2, respectively. The dominant phylotype,

264 representing $\sim 94 \%$ of the KSB3 reads, was identical to the previously reported bulking phylotype

265 (clone YM-1, AB218870; Yamada et al., 2007), and the minor phylotype, representing $\sim 6 \%$ of the

266 KSB3 reads, was identical to a low abundance clone detected in the UASB reactor during normal

267 operation (clone SmB78fl, AB266927; Narihiro et al., 2009). The internal transcribed spacer 
268 (ITS) region of the bulking phylotype was sequenced to confirm that it was the same strain 269 present in the bulking and normally operating UASB sludge (Supplementary Fig. S2).

270 Previously reported metagenomes (Soo et al., 2014) and additional shotgun sequencing of

271 samples A1, F1, G1 and A2 were used for recovering high quality draft population genomes of

272 the two KSB3 phylotypes (Supplementary Table S1). Based on detection of 16S rRNA genes in

273 the shotgun paired-end read datasets, the KSB3 phylotypes comprised up to 10 and $11 \%$ of the

274 A1 and A2 metagenomes respectively with the dominant KSB3 phylotype having approximately

27510 fold higher abundance than the minor phylotype (Supplementary Fig. S3), broadly consistent 276 with the amplicon results.

\section{Recovery of KSB3 population genomes}

278 The four metagenomes (59 Gb in total, Supplementary Table S1) were co-assembled, 279 generating 504,757 contigs/scaffolds (>500 bp) with a combined length of $906 \mathrm{Mb}$, an N50 of 3

$280 \mathrm{~kb}$ and a longest scaffold of $506 \mathrm{~kb}$. Population genomes were recovered from the assembly by 281 exploiting variations in population abundance (coverage) between individual sample

282 metagenomes (differential coverage binning, Albertsen et al., 2013) using the automated binning

283 tool GroopM (Imelfort et al., 2014). The completeness and contamination of the population

284 genomes were estimated by detection of single copy marker gene sets widely conserved in the 285 domain Bacteria (Dupont et al., 2012). Thirty-nine bacterial population genome bins were 286 obtained with $>65 \%$ completeness $(>73 / 111$ markers) and $<10 \%$ contamination $(<11 / 111$, marker

287 genes with $>1$ copy in a population genome indicate presumptive contamination with another 288 organism). These genomes were refined by tracking mate-pair reads in network graphs to further

289 improve the completeness and reduce contamination of the bins, and to recruit repeat sequences, 290 notably ribosomal RNA operons, which can evade differential coverage binning if present in 291 multiple copies (Albertsen et al., 2013). 
292 We identified 16S rRNA gene sequences in two refined population genome bins (UASB14 and

293 UASB270, Supplementary Fig. S4) that were identical to the amplicon sequences from the

294 dominant and minor KSB3 phylotypes, respectively (Table 1). Despite careful manual curation of

295 both genomes, their estimated completeness based on 111 conserved single copy marker genes

296 (Dupont et al., 2012) is only $\sim 93 \%$, and both also have an inferred $\sim 6 \%$ contamination based on

297 these markers. Inspection of the marker genes with no or $>1$ hit, however, show a high degree of

298 overlap between the genomes suggesting that these particular genes are either actually absent,

299 duplicated, or laterally transferred based on phylogenetic inference and gene neighborhood

300 (Supplementary Table S2). This may not be unexpected given the phylogenetic novelty of the

301 lineage. A revised estimate of completeness and contamination based on a prediction that six of 302 the 111 marker genes are absent and five are duplicated is $>98 \%$ and $<2 \%$ respectively (Table 1).

303 To estimate the number of rRNA operons in each KSB3 genome, we compared average genomic 304 coverage to $16 \mathrm{~S}$ rRNA gene coverage, which indicated that UASB14 and UASB270 have three 305 and two rRNA operons respectively (Supplementary Fig. S5).

\section{KSB3 phylogeny and morphology}

307 The relative position of the two KSB3 genomes within the phylum was assessed by comparative 308 analysis of their 16S rRNA gene sequences with publicly available full-length sequences.

309 UASB14 and UASB270 represent two of several major lines of descent in the KSB3 phylum

310 (Fig. 1a). According to Greengenes classification (McDonald et al., 2012), UASB14 belongs to

311 an unnamed class-level lineage and UASB270 is a member of class MAT-CR-H3-D11 for which

312 we propose the names Moduliflexia and Vecturitrichia, respectively (Table 1; Supplementary

313 Notes). To confirm the status of KSB3 as a candidate phylum, as inferred by 16S rRNA

314 comparative analyses (Yamada et al., 2007, Supplementary Fig. S6), we constructed 
315 phylogenetic trees based on a larger genomic sampling. Two sets of marker genes broadly

316 conserved in all domains of life (38 markers) (Darling et al., 2014) or in Bacteria (83 markers)

317 (Soo et al., 2014) were obtained from the KSB3 genomes and up to 354 publicly available

318 reference genomes (Markowitz et al., 2014). Each gene family was independently aligned and

319 ambiguous and/or non-informative positions removed, and then the filtered alignments were

320 concatenated for maximum-likelihood inference. Four sets of outgroup configurations were used

321 including representatives of all major genomically sampled bacterial phyla (Supplementary

322 Table S3). The two KSB3 genomes form a robustly monophyletic group in all analyses, and did

323 not reproducibly affiliate with any other phyla (Fig. 2, Supplementary Fig. S7, Supplementary

324 Table S4), consistent with the original proposal that KSB3 is a candidate bacterial phylum

325 (Yamada et al., 2007). The average amino acid identity (AAI) between UASB14 and UASB270 is

$32660.3 \%$ (Supplementary Fig. S8), and supports their assignment to separate classes as it falls

327 within the range of known class-level AAI values (44-61\%, Konstantinidis \& Tiedje, 2005).

328 To confirm the filamentous morphology and relative abundance of the KSB3 phylotypes, we

329 designed 16S rRNA-targeted fluorescence in situ hybridization (FISH) probes specific at the

330 phylum and class level and combined them with previously applied KSB3-specific probes

331 (Yamada et al., 2007) (Supplementary Table S5). We detected only filamentous KSB3

332 morphotypes in the UASB sludge and these comprised the majority of observed filaments in

333 sample F1 (Fig. 1c-d). The relative abundance of the two KSB3 phylotypes inferred from both

334 amplicon and metagenome data was also consistent with FISH analyses, that is, filaments

335 belonging to the class Moduliflexia (presumably mostly UASB14) greatly outnumbered those

336 belonging to the Venturitrichia (presumably mostly UASB270) (Fig. 1b), noting that the two

337 filaments were indistinguishable by light microscopy alone (Fig. 1c-d). 


\section{General features of the KSB3 genomes}

339 Both KSB3 genomes are large by bacterial standards, $>7 \mathrm{Mb}$ (Supplementary Fig. S9) and have

340 median GC content, $\sim 50 \%$ (Table 1). Since UASB14 and UASB270 are not close relatives, large

341 genome size may be a characteristic feature of the KSB3 phylum or at least of the two classes

342 that they represent (Fig. 1a). A total of 5,989 and 7,048 open reading frames (ORFs) were

343 identified in the UASB14 and UASB270 genomes, respectively (Table 1). For both genomes,

344 approximately two thirds of the ORFs had a predicted function and the remaining third were

345 hypotheticals. Reciprocal BLASTP best matches between the predicted gene products of the two

346 KSB3 genomes indicate a shared set of 3,296 orthologs, representing approximately half of the

347 gene inventories in each genome. Included in this common set are conserved genes for

348 translation, nucleotide transport and metabolism, and construction of a diderm (Gram negative)

349 cell envelope including lipopolysaccharide synthesis (Supplementary Fig. S10). We identified a

350 full complement of rRNA and tRNA genes in UASB14, but not UASB270, which were likely

351 missed in the latter genome (Table 1; Supplementary Table S6). The UASB14 rRNA genes are

352 estimated to be present as three nearly identical operons (Supplementary Fig. S5) collapsed into

353 a single large repeat during the assembly process. A number of large clustered regularly

354 interspaced short palindromic repeats (CRISPR) were identified in both genomes (Table 1).

355 CRISPR, together with associated cas genes, constitute a recently described defense mechanism

356 against invading foreign DNAs and have been found in a majority of bacterial genera and most

357 Archaea (Sorek, Kunin \& Hugenholtz, 2008). A COG category analysis of the KSB3 genomes

358 indicates that both have high relative proportions of carbohydrate metabolism and transport $(\mathrm{G})$

359 and signal transduction $(\mathrm{T})$ relative to the bacterial average (Fig. 3). More detailed inferred 360 metabolic properties of the two KSB3 genomes are described below.

\section{Strictly fermentative metabolism}


362 Both KSB3 representatives have an incomplete tri-carboxylic acid (TCA) cycle and lack most

363 electron-transport chain complexes including terminal oxidases, indicating a strictly fermentative

364 metabolism (Fig. 4). They encode, however, both superoxide reductase and thioredoxin

365 reductase, suggesting oxidative stress tolerance. Both genomes have a large complement of

366 transporters and enzymes for importing and degrading complex and simple carbohydrates, which

367 can then be fed into a complete glycolysis (Embden-Meyerhof-Parnas) pathway (Fig. 4; COG

368 category G in Fig. 3). Both filaments also are likely capable of hydrolyzing polymers such as

369 cellulose and starch via a range of glycoside hydrolases (Supplementary Table S7). They

370 redundantly encode four different enzymes for converting pyruvate to acetyl-coenzyme A

371 (acetyl-CoA), namely pyruvate dehydrogenase, pyruvate-formate lyase, pyruvate ferredoxin

372 oxidoreductase, and pyruvate-flavodoxin oxidoreductase. Both generate adenosine triphosphate

373 (ATP) by converting acetyl-CoA to acetate via two enzymes (acetate kinase and phosphate

374 acetyltransferase) commonly found in general fermentative anaerobes (Mai \& Adams, 1996), in

375 addition to glycolysis. They may reoxidize NADH produced during glycolysis by converting

376 pyruvate to D-lactate and acetyl-CoA to ethanol (Fig. 4).

377 The KSB3 genomes possess a large complement of enzymes for conversion and transport of

378 amino acid and peptides (Fig. 4; COG category E in Fig. 3), including numerous proteases and

379 peptidases (Table 1). Peptide and amino acid degradation in the KSB3 filaments may produce

380 pyruvate, oxaloacetate, succinyl-CoA, and possibly propionyl CoA (Fig. 4). Notably, a complete

381 set of genes for the methylmalonyl CoA pathway was identified in UASB270, suggesting a role

382 in either amino acid degradation, propionate oxidation and/or propionate formation as a

383 fermentative end product in this organism. Some fermentative anaerobes are known to produce

384 hydrogen to scavenge excess electrons generated during metabolism (Sieber, McInerney \&

385 Gunsalus, 2012). In both KSB3 genomes, we identified several hydrogenase genes (Fig. 4). By 
386 examining domain structure and gene neighbourhoods (Supplementary Fig. S11), we predict

387 that some of these genes encode catalytic enzymes. This may permit them to engage in syntrophic

388 interactions with hydrogenotrophs, such as methanogens, in the sludge granules (Sieber et al.,

389 2012). However, based on FISH experiments highlighting KSB3 and archaeal cells, we did not

390 observe a close proximity between the two groups that would facilitate syntrophy (data not

391 shown). Some of the hydrogenase genes are located next to signal transduction genes raising the

392 possibility that they are involved in signal transduction and chemotaxis (Supplementary Fig.

393 S11).

\section{Sensory capabilities and motility}

395 One of the most striking features of the KSB3 genomes is the presence of extensive regulatory 396 networks, including two-component signal transduction systems (Table 1; Supplementary Table

397 S8). Signal transduction genes (COG category T) are among the highest represented categories in

398 both genomes (Fig. 3). Two-component systems respond to a broad range of extracellular and

399 intracellular signals, and play a role in many cellular processes including growth, motility, and

400 the cell cycle (Galperin, 2004; Skerker et al., 2005; Kirby, 2009). UASB14 and UASB270 encode

401135 and 114 putative transmembrane sensor proteins likely used for environmental signaling

402 (Galperin, 2004), and 131 and 116 putative response regulators containing CheY-like domains,

403 respectively (Supplementary Table S8). They each contain over 60 methyl-accepting proteins

404 and numerous Che-like chemotaxis proteins (Supplementary Table S8). Even when 405 compensating for their relatively large genome sizes, both KSB3 genomes possess high 406 proportions of environmental sensory networks compared to other sequenced bacterial and 407 archaeal genomes (Fig. 5; Supplementary Figs. S12 and S13; Supplementary Table S9). The 408 high representation of sensory components in the KSB3 genomes is on par with social 
409 Myxococcales such as Sorangium cellulosum and Stigmatella aurantiaca, both of which exhibit

410 complex, self-organizing behavior in response to environmental stimuli (Huntley et al., 2011).

411 Sensory capabilities are an important component of a bacterium's overall social "intelligence" or

412 social IQ (Ben-Jacob et al., 2004), a metric recently proposed based on the abundance of two-

413 component systems, transcription factors, defense mechanisms and transport systems (Sirota-

414 Madi et al., 2010). We determined that the KSB3 filaments have among the highest social IQ

415 scores of any sequenced bacterial and archaeal species to date, scoring particularly well in the

416 two-component and transport system categories (Supplementary Fig. S14). This suggests that

417 the filaments are sensitive to their surroundings and capable of adaptable behavior in response to

418 changes in their local environment. Key to this adaptability is motility. No genes for flagella

419 production were identified in either KSB3 genome, so to determine if KSB3 bacteria are indeed

420 motile, we observed filaments enriched from UASB granules by wet mount microscopy under a

421 range of conditions. KSB3-specific FISH of samples taken in parallel confirmed that the majority

422 of filaments in these samples were members of the KSB3 phylum (Fig. 1). Initially no motility

423 was observed, therefore based on the metabolic reconstruction of the KSB3 genomes, we added a

424 range of compounds (mostly simple sugars, see Methods section) to the edge of the microscope

425 slides to create a gradient that could be sensed by the filaments to stimulate a motility response.

426 We observed gliding motility at rates of between 20 to $30 \mu \mathrm{m} / \mathrm{min}$ only when a glucose or

427 maltose gradient was applied under conditions mimicking the UASB reactor operation

428 (Supplementary Movie 1). Both KSB3 genomes encode a number of the genes necessary for

429 type IV pili formation (pilB, pilC, pilG, pilT, pilV, and flp pilus assembly protein) that may

430 enable gliding via extension and retraction (Jarrell \& McBride, 2008). However, the full gene

431 complement for pili formation (Mauriello et al., 2010) was not detected and the mechanism for

432 KSB3 gliding motility remains to be determined. 


\section{Discussion}

434 Despite the biotechnological significance of industrial-scale anaerobic digestion, our

435 understanding of the microbial ecology that underpins these processes is still rudimentary

436 because most microorganisms cannot be cultured and such systems are essentially managed as

437 "black boxes" (Ahring, 2003b; Rivière et al., 2009). Emerging culture-independent molecular

438 techniques such as differential coverage binning of metagenomic data, which allows even low

439 abundance population genomes to be recovered (Sharon et al., 2013; Albertsen et al., 2013), are

440 providing new opportunities to understand and optimize system performance (Vanwonterghem et

441 al., 2014).

442 Using this approach, we obtained the first population genomes representing candidate bacterial

443 phylum KSB3 (Tanner et al., 2000; Yamada et al., 2007). One of these genomes, UASB14,

444 belongs to a high abundance filament ( $10 \%$ of the community; Table 1; Fig. 1) previously

445 reported to be responsible for bulking in an industrial UASB system treating wastewater from

446 sugar manufacture (Yamada et al., 2007). A second genome from the same habitat, UASB270,

447 represents a low abundance $(<0.5 \%)$ filament only moderately related to the first, i.e. they

448 represent different classes within the KSB3 phylum (Fig. 1). Metabolic reconstruction indicates

449 that both filaments are primary fermenters of sugar and amino acid-containing compounds in the

450 system (Fig. 4), and both have a high "social IQ" based in part on possession of extensive

451 regulatory networks (Table 1; Supplementary Tables S8 and S9; Supplementary Fig. S14).

452 These findings support the hypothesis that KSB3 filaments are important primary fermenters in

453 healthy sludge granules (Yamada et al., 2011) and further suggest that the filaments are sensitive

454 to their surroundings and that their cellular processes, such as growth, may be controlled by

455 external signals. Whether these features can be extrapolated to the whole KSB3 phylum, or 
456 simply reflect the specialized habitat from which the genomes were obtained, remains to be

457 determined. Environmental surveys suggest that the phylum has a shallow ecological footprint,

458 having been identified in mostly anoxic saline habitats (Fig. 1a), which may indicate that a

459 fermentative metabolism is universal.

460 The inferred capacity of the filaments to detect physicochemical gradients in their surroundings

461 suggests that they should be motile. Apart from an incomplete gene complement for Type IV pili,

462 no motility mechanism could be identified. However, microscopic observations indicated that the

463 KSB3 filaments are capable of gliding motility in response to applied sugar gradients

464 (Supplementary Movie 1). Gliding motility is thought to have evolved independently in multiple

465 bacterial lineages, and the molecular mechanisms of gliding are only partially elucidated for a

466 limited number of bacterial taxa (Jarrell \& McBride, 2008; Mignot \& Kirby, 2008). This is the

467 first report of gliding motility of organisms in UASB sludge granules, which have long been

468 considered to have an organization driven by growth and attachment rather than motility of cells

469 (Liu et al., 2003; Hulshoff Pol et al., 2004). An enhanced sensory system is also likely the key

470 driver of the bulking phenomenon, that is, changes in the UASB reactor such as increases in

471 glucose or maltose concentration trigger outgrowth of the KSB3 filaments (Yamada et al., 2011).

472 It may also explain why repeated attempts to cultivate KSB3 filaments have failed to date

473 (Yamada et al., 2011), because they require specific and possibly complex environmental cues to

474 stimulate growth in axenic culture.

475 The inference that the KSB3 filaments sense sugars and the observation of a gliding motility 476 response in the presence of a glucose or maltose gradient is consistent with the previous 477 observation of uptake of these sugars by KSB3 filaments (Yamada et al., 2011). Plant operators

478 began monitoring glucose concentration in the UASB reactor influent using a simple urine test 
479 strip. No further bulking has occurred to date since keeping influent glucose concentration

480 uniformly low $(<200 \mathrm{mg} / \mathrm{L})$ via adjustment of retention times in the acidification pretreatment. A

481 more detailed understanding of environmental stimuli responsible for growth and bulking will be

482 facilitated by the availability of the KSB3 genome sequences which may lead to genome-directed

483 cultivation (Tyson et al., 2005) and other treatment options for bulking.

484 We propose the names 'Candidatus Moduliflexus flocculans' and 'Candidatus Vecturithrix 485 granuli' for the two KSB3 filament types represented by the UASB14 and UASB270 genomes 486 respectively, and the phylum name, Modulibacteria, and intermediate rank names (Table 1;

\section{Supplementary Notes).}

\section{Conclusions}

489 In summary, this study adds novel genomic 'foliage' to the tree of life by reporting the near 490 complete genomes of two phylogenetically diverse members of candidate bacterial phylum KSB3

491 obtained from an industrial UASB system. Genome-based metabolic reconstruction and

492 experimental observations provide clues to the roles of the KSB3 bacteria in the treatment system

493 including their ability to ferment sugars and chemotactically respond to glucose and maltose 494 gradients, laying the foundations for a detailed understanding of their ecophysiology and role in 495 wastewater bulking.

496 Accession codes. The genome sequences of 'Candidatus Moduliflexus flocculans' and 497 'Candidatus Vecturithrix granuli' have been deposited in DDBJ/EMBL/GenBank under the 498 accession numbers DF820455-DF820462 and DF820463-DF820483. The ITS sequences of the 499 two genotypes have also been deposited in the databases under the accession numbers AB933567 500 and $\mathrm{AB} 933568$. 


\section{Acknowledgments}

502 We thank Jason Steen, Ben Woodcroft, Mohamed F. Haroon, and Michael Imelfort from the

503 University of Queensland for assistance and advice on the bioinformatic analyses and Taeko

504 Yokoi from AIST for assistance with ITS and FISH experiments. We also thank Satoshi Hanada 505 from AIST and Bernhard Schink from the University of Konstanz for etymological advice.

\section{Author contributions}

507 Y.S., experimental design, data analysis and manuscript; A.O., molecular biology work and 508 sequencing; D.H.P. bioinformatic analyses; T.Y., bioreactor operation and sample collection; 509 G.W.T., data analysis and manuscript; P.H., data analysis and manuscript.

\section{Competing interests}

511 The authors declare no competing financial or other interests.

\section{Figure and table legends}

513 Figure 1 Phylogenetic structure of the Modulibacteria (KSB3) phylum based on comparative

514 analysis of 16S rRNA gene sequences, and imaging of KSB3 cells. (a) Maximum-likelihood

515 phylogenetic tree (RAxML) of public data (accession numbers shown) and the 16S rRNA

516 sequence determined in this study for UASB14. Sequences from the bacterial phyla Nitrospirae,

517 Tenericutes, and Chloroflexi were used to root the tree (not shown). Reproducible interior nodes

518 are indicated as a black circle ( $>90 \%$ bootstrap support for neighbor-joining [NJ], maximum

519 parsimony [MP], and maximum-likelihood [ML] inferences), open circle (>80\% support); or

520 open rectangle ( $>70 \%$ support). Nodes without symbols were not reproducible between trees. The

521 scale bar represents 5\% estimated sequence divergence. Class-level clades are bracketed to the 
522 right of the figure in black. The target ranges of KSB3-specific FISH (fluorescence in situ

523 hybridization) probes used in this study are indicated by colored brackets with the colors

524 corresponding to cell color in panels b and d. (b) 16S rRNA-targeted FISH detection of UASB14

525 and UASB270 filaments in the UASB sludge. The abundant UASB14 filaments are labeled green

526 and the low abundance UASB270 filaments are labeled red. (c) Total KSB3 filament abundance

527 highlighted by a phylum-level FISH probe relative to (d) all cells present in the same field

528 (phase-contrast image). Bars in panels b-d represent $10 \mu \mathrm{m}$.

529 Figure 2 Maximum-likelihood phylogenetic inference of Modulibacteria (KSB3) population 530 genomes among known bacterial phyla. The tree was constructed using RAxML based on up to

53138 marker genes (using taxon-outgroup configuration Config 3, Supplementary Table S3) and

532 sequences were collapsed at the phylum level except for classes in the Proteobacteria. Ranks are

533 indicated by prefix; $p_{\text {_ }}$ (phylum), c_ (class). KSB3 genomes obtained in this study are

534 highlighted in red. Superphyla (Terrabacteria, Patescibacteria, Fibrobacteres-Chlorobi-

535 Bacteroidetes [FCB], and Planctomycetes-Verrucomicrobia-Chlamydiae [PVC]) are highlighted

536 with color ranges. Taxa comprising cultivated representatives are shown in black; taxa with no

537 cultivated representatives are indicated by outline. Reproducible associations ( $>80 \%$ bootstrap

538 values from 100 resamplings) are indicated by dots on interior nodes. Alignments of homologous

539 proteins from archaeal genomes were used to root the tree (not shown). The scale bar represents

$54010 \%$ estimated sequence divergence.

541 Figure 3 Relative representation of COG categories by predicted ORFs in the UASB14 and

542 UASB270 genomes. Global averages and standard deviation (bars) are shown for 2279 publicly

543 available finished bacterial and archaeal genomes (Markowitz et al., 2014). Statistically 
544 significant differences are indicated by percentile of scores for all the available finished bacterial

545 and archaeal genomes.

546 Figure 4 Composite metabolic overview of the Modulibacteria (KSB3) genomes based on

547 identified genes and pathways. Gray indicates elements common to both genomes, while orange

548 and green show elements specific to UASB14 and UASB270, respectively. Both filament types

549 have the genes necessary to produce acetate, ethanol, lactate, and hydrogen (and possibly

550 propionate) as fermentative end products, likely generating energy through the glycolytic

551 Embden-Meyerhof-Parnas (EMP) pathway and the fermentation of amino acids and sugars.

552 Abbreviations; ETF, electron transfer flavoprotein; Fd-ox and Fd-red, oxidized and reduced

553 ferredoxin, respectively; UQ, ubiquinone.

554 Figure 5 Number of protein domains inferred to be involved in environmental signaling

555 (Supplementary Table S8) as a function of genome size for the two Modulibacteria (KSB3)

556 genomes (in red) and 2279 publicly available finished bacterial and archaeal genomes (in blue).

557 The KSB3 filaments have among the highest proportion of signaling domains, only surpassed by

558 members of the Myxobacteria (open blue circles), which are capable of fruiting body formation

559 by contact-mediated signaling.

560 Table 1 Features of the improved high-quality KSB3 ('Modulibacteria') population genomes.

561 Additional File 1 Supplementary Notes, Supplementary Figs and Tables.

562 Additional File 2 Supplementary Movie 1. Time-lapse video microscopy showing gliding

563 motility of KSB3-like filaments in a UASB aggregate. The aggregate was exposed to a glucose 
564 gradient under anaerobic conditions at $37^{\circ} \mathrm{C}$. This video is shown at $45 \times$ real time. 
References

566

567

568

569

570

571

572

573

574

575

576

577

578

579

580

581

582

583

584

585

586

587

588

589

590

591

592

593

594

595

596

Ahring BK 2003a. Biomethanation I. Springer.

Ahring BK 2003b. Perspectives for Anaerobic Digestion. In: Biomethanation I. Advances in Biochemical Engineering/Biotechnology. Berlin, Heidelberg: Springer, 1-30.

Albertsen M, Hugenholtz P, Skarshewski A, Nielsen KL, Tyson GW, Nielsen PH 2013. Genome sequences of rare, uncultured bacteria obtained by differential coverage binning of multiple metagenomes. Nature Biotechnology 31:533-538.

Angelidaki I, Karakashev D, Batstone DJ, Plugge CM, Stams AJM 2011. Biomethanation and its potential. Methods in Enzymology 494:327-351.

Aoki-Kinoshita KF, Kanehisa M 2007. Gene annotation and pathway mapping in KEGG. Methods in Molecular Biology 396:71-91.

Ben-Jacob E, Becker I, Shapira Y, Levine H. 2004. Bacterial linguistic communication and social intelligence. Trends Microbiol 12:366-372.

Bland C, Ramsey TL, Sabree F, Lowe M, Brown K, Kyrpides NC, Hugenholtz P 2007. CRISPR Recognition Tool (CRT): a tool for automatic detection of clustered regularly interspaced palindromic repeats. BMC Bioinformatics 8:209.

Boetzer M, Henkel CV, Jansen HJ, Butler D, Pirovano W 2011. Scaffolding pre-assembled contigs using SSPACE. Bioinformatics 27:578-579.

Caporaso JG, Bittinger K, Bushman FD, Desantis TZ, Andersen GL, Knight R 2010a. PyNAST: a flexible tool for aligning sequences to a template alignment. Bioinformatics 26:266-267.

Caporaso JG, Kuczynski J, Stombaugh J, Bittinger K, Bushman FD, Costello EK, Fierer N, Peña AG, Goodrich JK, Gordon JI, Huttley GA, Kelley ST, Knights D, Koenig JE, Ley RE, Lozupone CA, McDonald D, Muegge BD, Pirrung M, Reeder J, Sevinsky JR, Turnbaugh PJ, Walters WA, Widmann J, Yatsunenko T, Zaneveld J, Knight R. 2010b. QIIME allows analysis of high-throughput community sequencing data. Nature Methods 7:335-336.

Caporaso JG, Lauber CL, Walters WA, Berg-Lyons D, Huntley J, Fierer N, Owens SM, Betley J, Fraser L, Bauer M, Gormley N, Gilbert JA, Smith G, Knight R. 2012. Ultra-high-throughput microbial community analysis on the Illumina HiSeq and MiSeq platforms. The ISME Journal 6:1621-1624.

Castelle CJ, Hug LA, Wrighton KC, Thomas BC, Williams KH, Wu D, Tringe SG, Singer SW, Eisen JA, Banfield JF 2013. Extraordinary phylogenetic diversity and metabolic versatility in aquifer sediment. Nature Communications 4:2120. 
597 Darling AE, Jospin G, Lowe E, Matsen FA IV, Bik HM, Eisen JA 2014. PhyloSift: phylogenetic 598 analysis of genomes and metagenomes. PeerJ 2:e243.

599 Doetsch RN 1981. Determinative methods of light microscopy. In: Gerhardt P ed. Manual of 600 methods for general bacteriology. Manual of methods for general bacteriology.

601 Dupont CL, Rusch DB, Yooseph S, Lombardo M-J, Richter RA, Valas R, Novotny M, Yee602 Greenbaum J, Selengut JD, Haft DH, Halpern AL, Lasken RS, Nealson K, Friedman R, Venter JC. 2012. Genomic insights to SAR86, an abundant and uncultivated marine bacterial

605 Eddy SR 2011. Accelerated profile HMM searches. PLoS Computational Biology 7:e1002195.

606 Felsenstein J 1989. PHYLIP - Phylogeny inference package (version 3.2). Cladistics 5:164-166.

607 Galperin MY 2004. Bacterial signal transduction network in a genomic perspective. 608 Environmental Microbiology 6:552-567.

609 Hulshoff Pol LW, de Castro Lopes SI, Lettinga G, Lens PNL 2004. Anaerobic sludge granulation. $610 \quad$ Water Research 38:1376-1389.

611 Huntley S, Hamann N, Wegener-Feldbrügge S, Treuner-Lange A, Kube M, Reinhardt R, Klages 612 S, Müller R, Ronning CM, Nierman WC, Søgaard-Andersen L. 2011. Comparative genomic 613 analysis of fruiting body formation in Myxococcales. Molecular Biology and Evolution 614 28:1083-1097.

615 Imelfort M, Parks DH, Woodcroft BJ, Dennis P, Hugenholtz P, Tyson GW. 2014 GroopM: an 616 automated tool for the recovery of population genomes from related metagenomes. PeerJ $6172: \mathrm{e} 603$.

618 Jarrell KF, McBride MJ 2008. The surprisingly diverse ways that prokaryotes move. Nature 619 Reviews Microbiology 6:466-476.

620 Kirby JR 2009. Chemotaxis-like regulatory systems: unique roles in diverse bacteria. Annual 621 Reviews in Microbiology 63:45-59.

622 Kleerebezem R, Macarie H 2003. Treating industrial wastewater: anaerobic digestion comes of 623 age. Chemical Engineering 110:56-64.

624 Konstantinidis KT, Tiedje JM 2005. Towards a genome-based taxonomy for prokaryotes. Journal 625 of Bacteriology 187:6258-6264.

626 Krzywinski M, Schein J, Birol I, Connors J, Gascoyne R, Horsman D, Jones SJ, Marra MA 2009.

627 Circos: an information aesthetic for comparative genomics. Genome Research 19:16396281645 . 
629 Letunic I, Bork P 2011. Interactive Tree Of Life v2: online annotation and display of 630 phylogenetic trees made easy. Nucleic Acids Research 39:W475-8.

631 Li H, Durbin R 2010. Fast and accurate long-read alignment with Burrows-Wheeler transform. 632 Bioinformatics 26:589-595.

633 Li J, Hu B, Zheng P, Qaisar M, Mei L 2008. Filamentous granular sludge bulking in a laboratory 634 scale UASB reactor. Bioresource Technology 99:3431-3438.

635 Liu Y, Xu H-L, Yang S-F, Tay J-H 2003. Mechanisms and models for anaerobic granulation in 636 upflow anaerobic sludge blanket reactor. Water Research 37:661-673.

637 Lombard V, Ramulu HG, Drula E, Coutinho PM, Henrissat B 2014. The carbohydrate-active 638 enzymes database (CAZy) in 2013. Nucleic Acids Research 42:D490-D495.

639 Ludwig W, Strunk O, Westram R, Richter L, Meier H, Yadhukumar, Buchner A, Lai T, Steppi S, 640 Jobb G, Förster W, Brettske I, Gerber S, Ginhart AW, Gross O, Grumann S, Hermann S, Jost 641 R, König A, Liss T, Lüssmann R, May M, Nonhoff B, Reichel B, Strehlow R, Stamatakis A, 642 Stuckmann N, Vilbig A, Lenke M, Ludwig T, Bode A, Schleifer K-H. 2004. ARB: a software 643 environment for sequence data. Nucleic Acids Research 32:1363-1371.

644 Mai X, Adams MW 1996. Purification and characterization of two reversible and ADP-dependent 645 acetyl coenzyme A synthetases from the hyperthermophilic archaeon Pyrococcus furiosus. 646 Journal of Bacteriology 178:5897-5903.

647 Marchler-Bauer A, Zheng C, Chitsaz F, Derbyshire MK, Geer LY, Geer RC, Gonzales NR, 648 Gwadz M, Hurwitz DI, Lanczycki CJ, Lu F, Lu S, Marchler GH, Song JS, Thanki N, 649 Yamashita RA, Zhang D, Bryant SH. 2013. CDD: conserved domains and protein three650 dimensional structure. Nucleic Acids Research 41:D348-52.

651 Markowitz VM, Chen I-MA, Chu K, Szeto E, Palaniappan K, Pillay M, Ratner A, Huang J, 652 Pagani I, Tringe S, Huntemann M, Billis K, Varghese N, Tennessen K, Mavromatis K, Pati A, 653 Ivanova NN, Kyrpides NC. 2014. IMG/M 4 version of the integrated metagenome 654 comparative analysis system. Nucleic Acids Research 42:D568-73.

Mauriello EMF, Mignot T, Yang Z, Zusman DR 2010. Gliding motility revisited: how do the myxobacteria move without flagella? Microbiology and Molecular Biology Reviews 74:229249.

659

McDonald D, Price MN, Goodrich J, Nawrocki EP, Desantis TZ, Probst A, Andersen GL, Knight R, Hugenholtz P 2012. An improved Greengenes taxonomy with explicit ranks for ecological and evolutionary analyses of bacteria and archaea. The ISME Journal 6:610-618. 
661 Mignot T, Kirby JR 2008. Genetic circuitry controlling motility behaviors of Myxococcus 662 xanthus. BioEssays 30:733-743.

663 Moller S, Croning M, Apweiler R 2002. Evaluation of methods for the prediction of membrane 664 spanning regions. Bioinformatics 18:218.

665 Narihiro T, Terada T, Kikuchi K, Iguchi A, Ikeda M, Yamauchi T, Shiraishi K, Kamagata Y, 666 Nakamura K, Sekiguchi Y 2009. Comparative analysis of bacterial and archaeal communities in methanogenic sludge granules from upflow anaerobic sludge blanket reactors treating various food-processing, high-strength organic wastewaters. Microbes and Environments 24:88-96.

Parks DH, Imelfort M, Skennerton CT, Hugenholtz P, Tyson GW. 2014 CheckM: assessing the quality of microbial genomes recovered from isolates, single cells, and metagenomes. PeerJ PrePrints 2:e554v1 http://dx.doi.org/10.7287/peerj.preprints.554v1.

Price MN, Dehal PS, Arkin AP 2009. FastTree: computing large minimum evolution trees with profiles instead of a distance matrix. Molecular Biology and Evolution 26:1641-1650.

Punta M, Coggill PC, Eberhardt RY, Mistry J, Tate J, Boursnell C, Pang N, Forslund K, Ceric G, Clements J, Heger A, Holm L, Sonnhammer ELL, Eddy SR, Bateman A, Finn RD. 2012. The Pfam protein families database. Nucleic Acids Research 40:D290-301.

Quevillon E, Silventoinen V, Pillai S, Harte N, Mulder N, Apweiler R, Lopez R 2005. InterProScan: protein domains identifier. Nucleic acids research 33:W116-W120.

Rivière D, Desvignes V, Pelletier E, Chaussonnerie S, Guermazi S, Weissenbach J, Li T, Camacho P, Sghir A 2009. Towards the definition of a core of microorganisms involved in anaerobic digestion of sludge. The ISME Journal 3:700-714.

Seemann T 2014. Prokka: rapid prokaryotic genome annotation. Bioinformatics 30: 2068-2069.

691 Sekiguchi Y, Kamagata Y, Nakamura K, Ohashi A, Harada H 1999. Fluorescence in situ hybridization using $16 \mathrm{~S}$ rRNA-targeted oligonucleotides reveals localization of methanogens and selected uncultured bacteria in mesophilic and thermophilic sludge granules. Applied and Environmental Microbiology 65:1280-1288.

Shannon P, Markiel A, Ozier O, Baliga NS, Wang JT, Ramage D, Amin N, Schwikowski B, Ideker T 2003. Cytoscape: a software environment for integrated models of biomolecular interaction networks. Genome Research 13:2498-2504.

Sharon I, Morowitz MJ, Thomas BC, Costello EK, Relman DA, Banfield JF 2013. Time series community genomics analysis reveals rapid shifts in bacterial species, strains, and phage during infant gut colonization. Genome Research 23:111-120. 
694 Sieber JR, McInerney MJ, Gunsalus RP 2012. Genomic insights into syntrophy: the paradigm for 695 anaerobic metabolic cooperation. Annual Review of Microbiology 66:429-452.

696 Sirota-Madi A, Olender T, Helman Y, Ingham C, Brainis I, Roth D, Hagi E, Brodsky L, 697 Leshkowitz D, Galatenko V, Nikolaev V, Mugasimangalam RC, Bransburg-Zabary S, 698 Gutnick DL, Lancet D, Ben-Jacob E. 2010. Genome sequence of the pattern forming 699 Paenibacillus vortex bacterium reveals potential for thriving in complex environments. BMC $700 \quad$ Genomics 11:710.

701 Skerker JM, Prasol MS, Perchuk BS, Biondi EG, Laub MT 2005. Two-component signal 702 transduction pathways regulating growth and cell cycle progression in a bacterium: a systemlevel analysis. PLoS Biology 3:e334.

704 Soo RM, Skennerton CT, Sekiguchi Y, Imelfort M, Paech SJ, Dennis PG, Steen JA, Parks DH, 705 Tyson GW, Hugenholtz P 2014. An expanded genomic representation of the phylum cyanobacteria. Genome Biology and Evolution 6:1031-1045.

707 Sorek R, Kunin V, Hugenholtz P 2008. CRISPR | a widespread system that provides acquired 708 resistance against phages in bacteria and archaea. Nature Reviews Microbiology 6:181-186.

709 Stamatakis A 2006. RAxML-VI-HPC: maximum likelihood-based phylogenetic analyses with 710 thousands of taxa and mixed models. Bioinformatics 22:2688-2690.

711 Suzek BE, Huang H, McGarvey P, Mazumder R, Wu CH 2007. UniRef: comprehensive and non712 redundant UniProt reference clusters. Bioinformatics 23:1282-1288.

713 Swofford DL 2003. PAUP*: phylogenetic analysis using parsimony, version 4.0b10.

714 Talavera G, Castresana J 2007. Improvement of phylogenies after removing divergent and 715 ambiguously aligned blocks from protein sequence alignments. Systematic Biology 56:564716577.

717 Tanner MA, Everett CL, Coleman WJ, Yang MM 2000. Complex microbial communities 718 inhabiting sulfide-rich black mud from marine coastal environments. Biotechnology et alia $7198: 1-16$.

720 Tatusov RL, Galperin MY, Natale DA, Koonin EV 2000. The COG database: a tool for genome721 scale analysis of protein functions and evolution. Nucleic Acids Research 28:33-36.

722 Tyson GW, Lo I, Baker BJ, Allen EE, Hugenholtz P, Banfield JF 2005. Genome-directed isolation 723 of the key nitrogen fixer Leptospirillum ferrodiazotrophum sp. nov. from an acidophilic 724 microbial community. Applied and Environmental Microbiology 71:6319-6324. 
725 van Lier JB 2008. High-rate anaerobic wastewater treatment: diversifying from end-of-the-pipe 726 treatment to resource-oriented conversion techniques. Water Science and Technology $727 \quad 57: 1137$.

728 Vanwonterghem I, Jensen PD, Ho DP, Batstone DJ, Tyson GW 2014. Linking microbial 729 community structure, interactions and function in anaerobic digesters using new molecular 730 techniques. Current Opinion in Biotechnology 27:55-64.

731 Yamada T, Sekiguchi Y 2009. Cultivation of uncultured Chloroflexi subphyla: significance and 732 ecophysiology of formerly uncultured Chloroflexi "subphylum I" with natural and 733 biotechnological relevance. Microbes and Environments 24:205-216.

734 Yamada T, Kikuchi K, Yamauchi T, Shiraishi K, Ito T, Okabe S, Hiraishi A, Ohashi A, Harada H, 735 Kamagata Y, Nakamura K, Sekiguchi Y. 2011. Ecophysiology of uncultured filamentous 736 anaerobes belonging to the phylum KSB3 that cause bulking in methanogenic granular 737 sludge. Applied and Environmental Microbiology 77:2081-2087.

738 Yamada T, Yamauchi T, Shiraishi K, Hugenholtz P, Ohashi A, Harada H, Kamagata Y, Nakamura 739 K, Sekiguchi Y 2007. Characterization of filamentous bacteria, belonging to candidate 740 phylum KSB3, that are associated with bulking in methanogenic granular sludges. The ISME 741 Journal 1:246-255.

742 Yin Y, Mao X, Yang J, Chen X, Mao F, Xu Y 2012. dbCAN: a web resource for automated 743 carbohydrate-active enzyme annotation. Nucleic Acids Research 40:W445-51. 
Figure 1: Phylogenetic structure of the Modulibacteria (KSB3) phylum based on comparative analysis of 16S rRNA gene sequences, and imaging of KSB3 cells.

(a) Maximum-likelihood phylogenetic tree (RAxML) of public data (accession numbers shown) and the 16S rRNA sequence determined in this study for UASB14. Sequences from the bacterial phyla Nitrospirae, Tenericutes, and Chloroflexi were used to root the tree (not shown). Reproducible interior nodes are indicated as a black circle ( $>90 \%$ bootstrap support for neighbor-joining [NJ], maximum parsimony [MP], and maximum-likelihood [ML] inferences), open circle (>80\% support); or open rectangle (>70\% support). Nodes without symbols were not reproducible between trees. The scale bar represents $5 \%$ estimated sequence divergence. Class-level clades are bracketed to the right of the figure in black. The target ranges of KSB3-specific FISH (fluorescence in situ hybridization) probes used in this study are indicated by colored brackets with the colors corresponding to cell color in panels $b$ and d. (b) 16S rRNA-targeted FISH detection of UASB14 and UASB270 filaments in the UASB sludge. The abundant UASB14 filaments are labeled green and the low abundance UASB270 filaments are labeled red. (c) Total KSB3 filament abundance highlighted by a phylum-level FISH probe relative to (d) all cells present in the same field (phase-contrast image). Bars in panels b-d represent $10 \mu \mathrm{m}$. 
a

$$
\begin{aligned}
& ->90 \text { (all NJ, MP, ML) } \\
& 0>80 \text { (all NJ, MP, ML) } \\
& \square>70 \text { (all NJ, MP, ML) }
\end{aligned}
$$
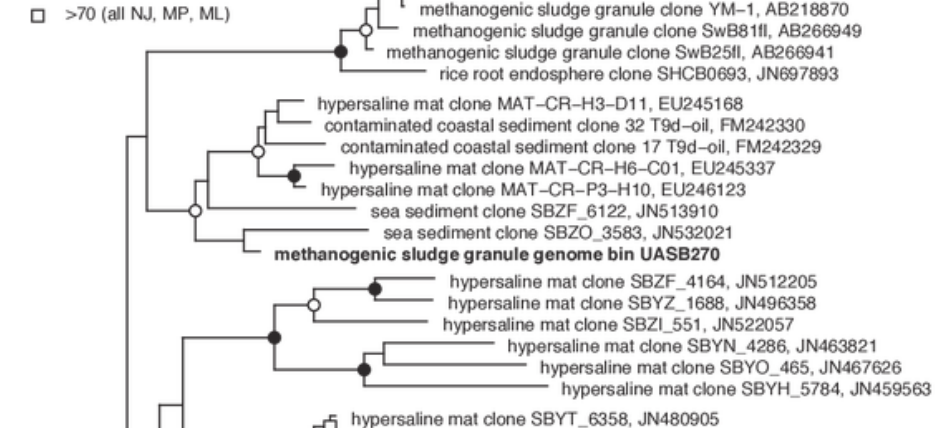

C hypersaline mat clone SBYT_6358, JN480905

- hypersaline mat done SBYB 3269, JN440125

- hypersaline mat clone SBYH_6792, JN458054

- sea sediment clone KZNMV-5-B94, FJ712494

sea sediment clone KZNMV-10-B57, FJ712533

- sea sediment clone $\mathrm{Cm} 1-19$, GQ246356

sea sediment clone AMSMV-0-B52, HQ588360

- sea sediment clone B5-087, JN977386

- hypersaline mat clone SBZF_8020, JN514940

대 hypersaline mat clone SBYZ 1134, JN492431

hypersaline mat clone SBZP_4170, JN537487

hypersaline mat clone $062 \mathrm{DZ} 43, \mathrm{DQ} 329580$

hypersaline mat clone SBZC_6018, JN509527

hypersaline mat clone SBYN_4356, JN463883

hypersaline mat clone SBYX_4363, JN489148

hypersaline mat clone GNO1-8.076, DQ15483

- hypersaline mat clone SBYB_1566, JN438063

- spring sediment clone Group7_f, JN387314

spring sediment clone Group7 g. JN387315

hypersaline mat clone MAT-CR-H3-C05, EU245159.1, 1515 hypersaline mat clone SBZC_2442, JN506565

- terrestrial mud volcano clone SYNH02_C3-13B, JQ245576 sea sediment clone 55 O I 5 . FN396658

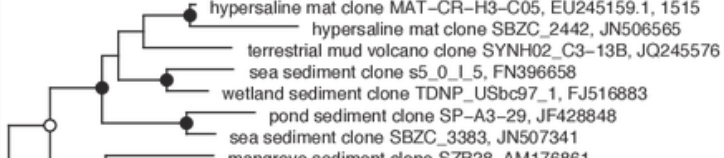

sea sediment clone SBZC_3383, JN507341

phreatic sinkhole clone Z100B62, FJ484060

tubeworm associated clone from cold seep v1141, FM165273

sea sediment clone GoC_Bac_115_D, FN820319

methane seep sediment clone Mn3b-B5, FJ264598

0.05

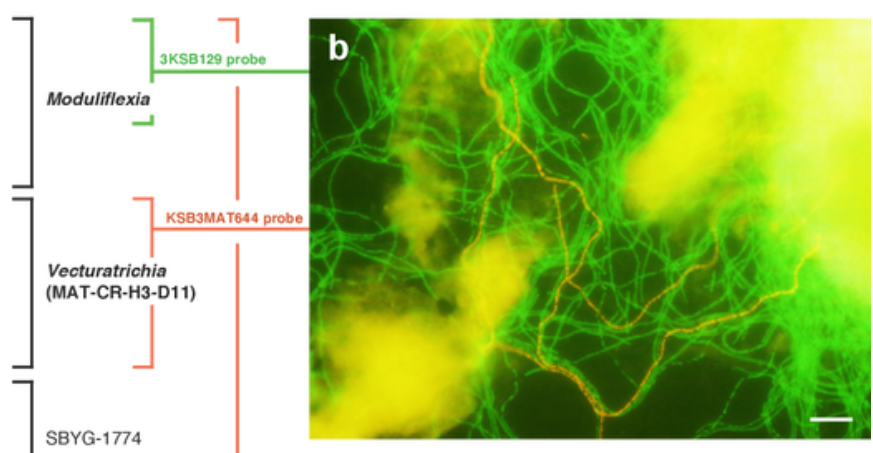

SBYG-1774

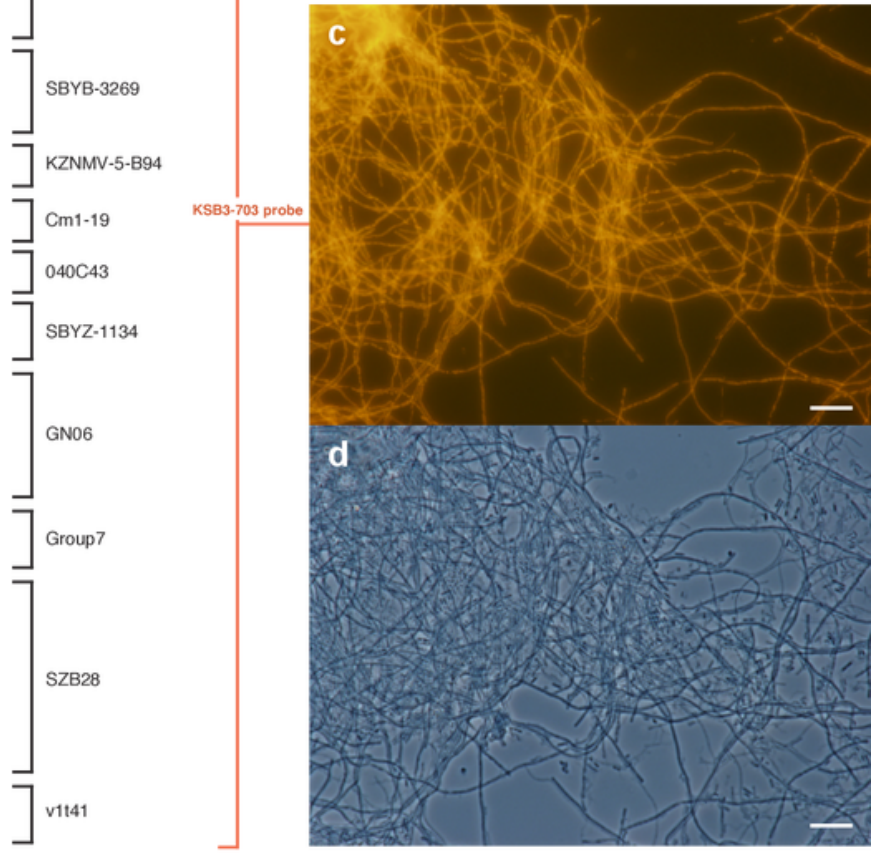




\section{2}

Figure 2: Maximum-likelihood phylogenetic inference of Modulibacteria (KSB3) population genomes among known bacterial phyla.

The tree was constructed using RAxML based on up to 38 marker genes (using taxonoutgroup configuration Config 3, Supplementary Table 3) and sequences were collapsed at the phylum level except for classes in the Proteobacteria. Ranks are indicated by prefix; $p_{-}$ (phylum), c_ (class). KSB3 genomes obtained in this study are highlighted in red. Superphyla (Terrabacteria, Patescibacteria, Fibrobacteres-Chlorobi-Bacteroidetes [FCB], and Planctomycetes-Verrucomicrobia-Chlamydiae [PVC]) are highlighted with color ranges. Taxa comprising cultivated representatives are shown in black; taxa with no cultivated representatives are indicated by outline. Reproducible associations ( $>80 \%$ bootstrap values from 100 resamplings) are indicated by dots on interior nodes. Alignments of homologous proteins from archaeal genomes were used to root the tree (not shown). The scale bar represents $10 \%$ estimated sequence divergence. 


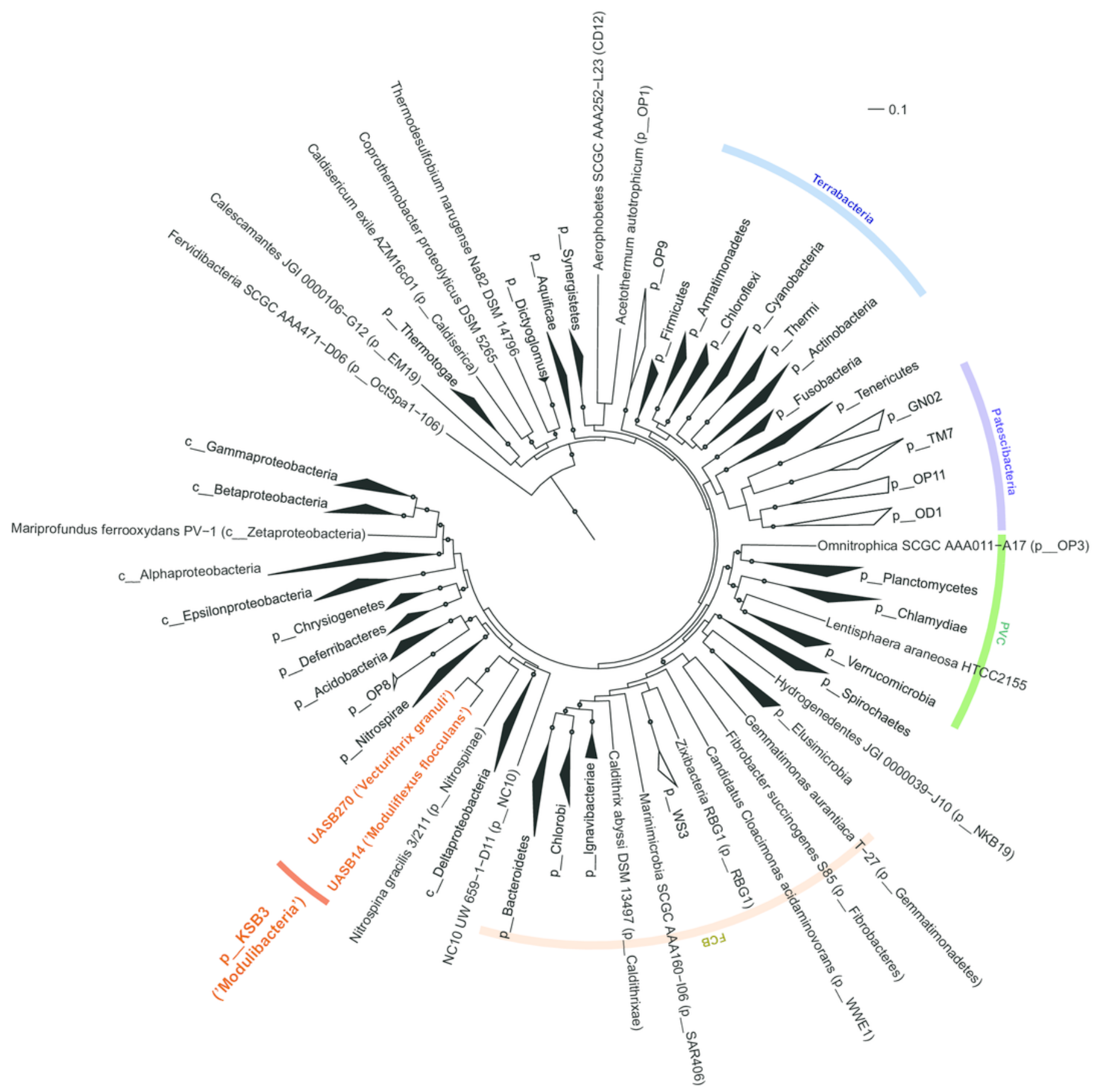


3

Figure 3: Relative representation of COG categories by predicted ORFs in the UASB14 and UASB270 genomes.

Global averages and standard deviation (bars) are shown for 2279 publicly available finished bacterial and archaeal genomes (Markowitz et al., 2014). Statistically significant differences are indicated by percentile of scores for all the available finished bacterial and archaeal genomes. 


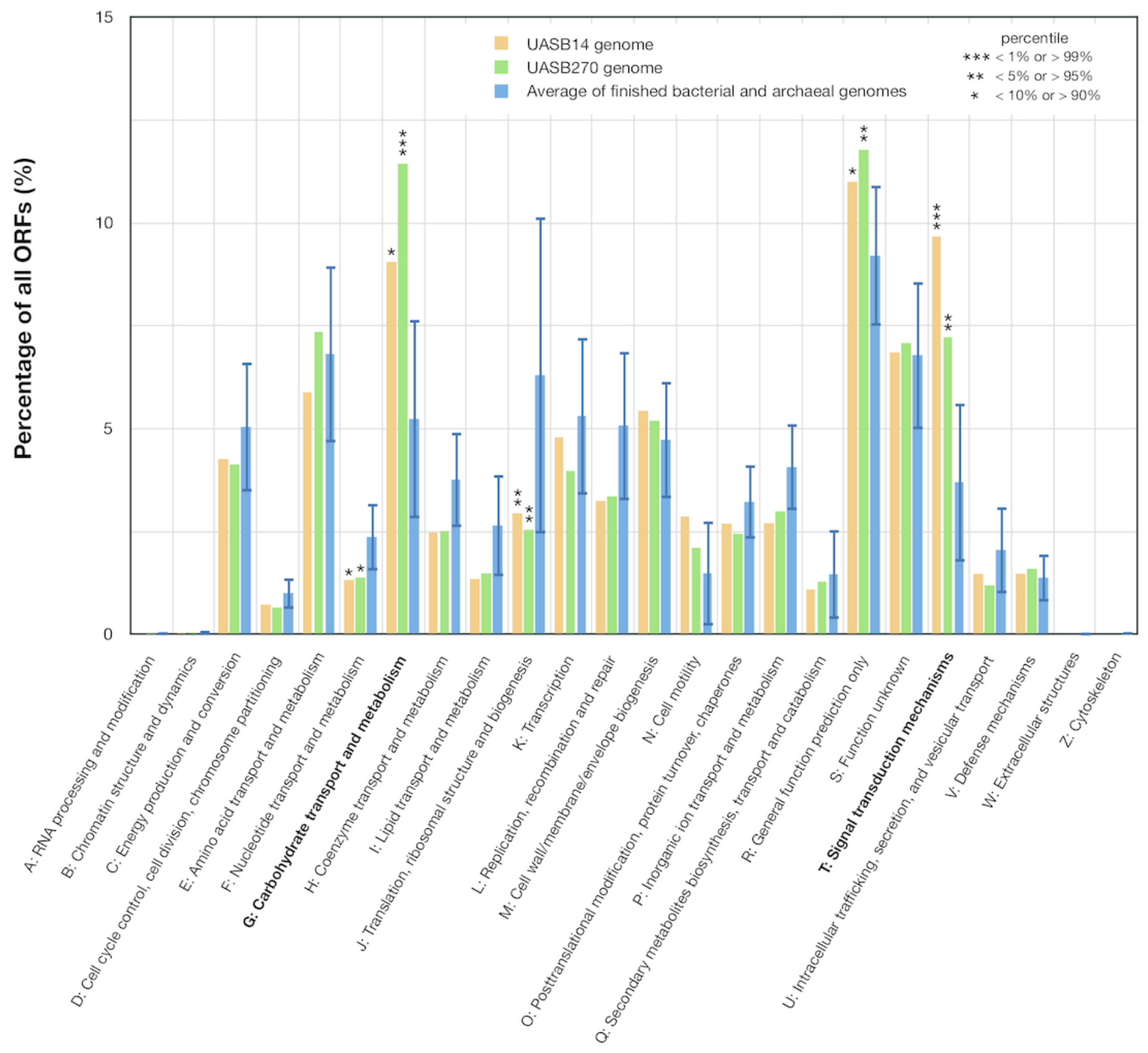

COG category 
Figure 4: Composite metabolic overview of the Modulibacteria (KSB3) genomes based on identified genes and pathways.

Gray indicates elements common to both genomes, while orange and green show elements specific to UASB14 and UASB270, respectively. Both filament types have the genes necessary to produce acetate, ethanol, lactate, and hydrogen (and possibly propionate) as fermentative end products, likely generating energy through the glycolytic EmbdenMeyerhof-Parnas (EMP) pathway and the fermentation of amino acids and sugars. Abbreviations; ETF, electron transfer flavoprotein; Fd-ox and Fd-red, oxidized and reduced ferredoxin, respectively; UQ, ubiquinone.

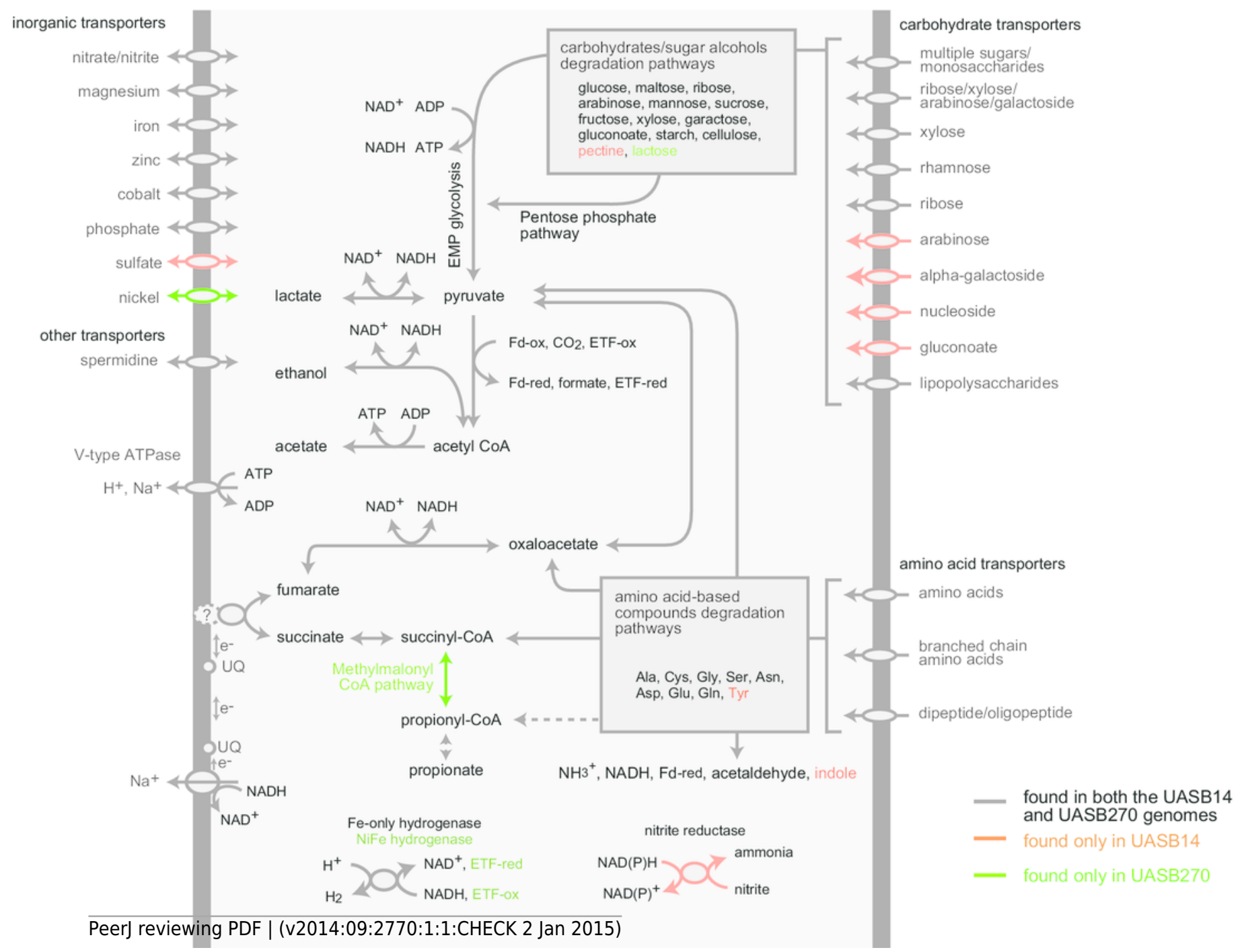




\section{5}

Figure 5: Number of protein domains inferred to be involved in environmental signaling for the two Modulibacteria (KSB3) genomes and finished bacterial and archaeal genomes.

Number of protein domains inferred to be involved in environmental signaling (Supplementary Table S8) as a function of genome size for the two Modulibacteria (KSB3) genomes (in red) and 2279 publicly available finished bacterial and archaeal genomes (in blue). The KSB3 filaments have among the highest proportion of signaling domains, only surpassed by members of the Myxobacteria (open blue circles), which are capable of fruiting body formation by contact-mediated signaling.

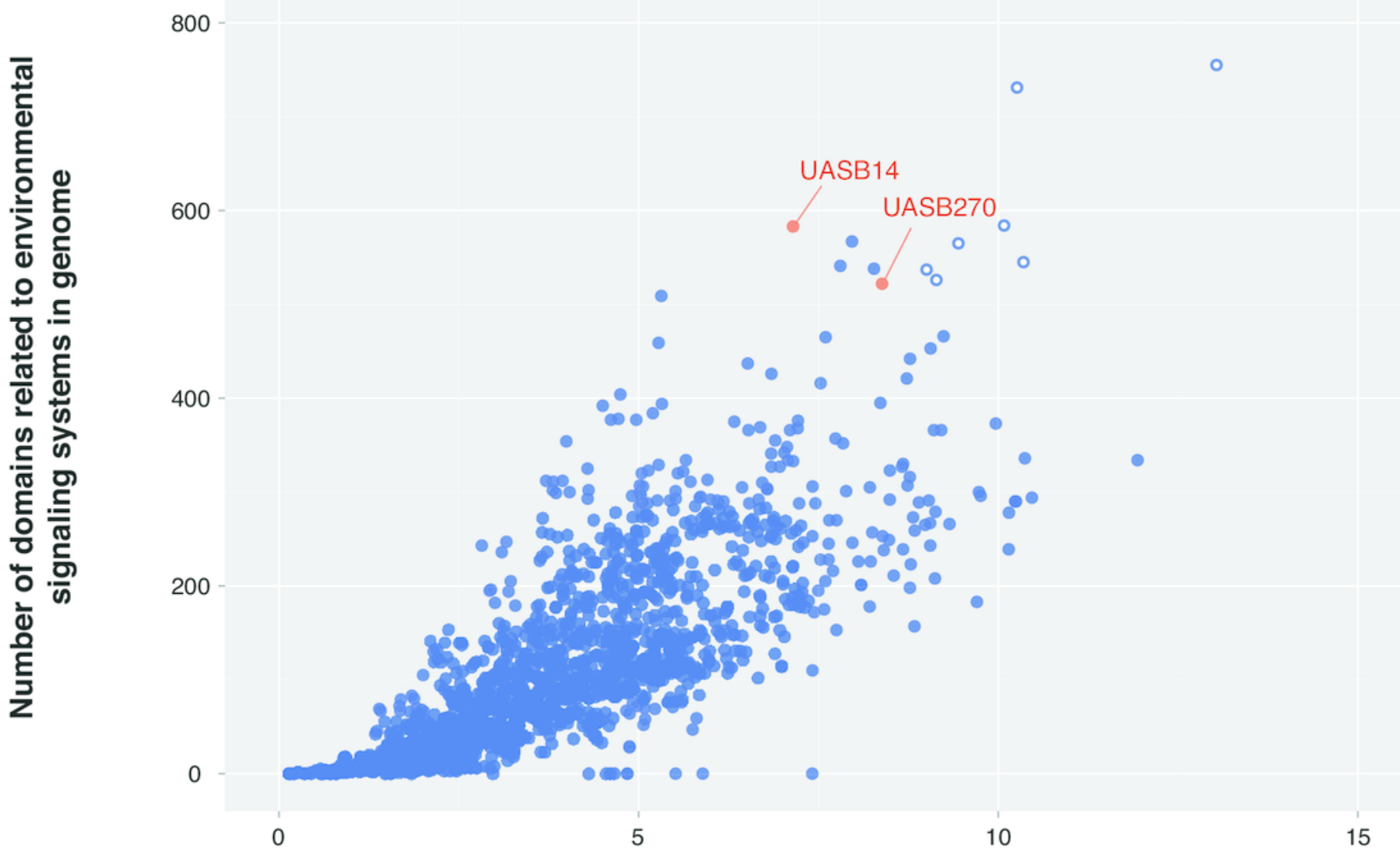

Genome size (Mb) 
Table $\mathbf{1}$ (on next page)

Table 1: Features of the Modulibacteria KSB3 population genomes. 
Table $1 \mid$ Features of the Modulibacteria KSB3 population genomes.

\begin{tabular}{|c|c|c|}
\hline Genome bin identifier & UASB14 & UASB270 \\
\hline Candidatus name & Moduliflexus flocculans & Vecturathrix granuli \\
\hline Closest environmental $16 \mathrm{~S}$ clone & YM-1 (AB218870) & SmB78fl (AB266927) \\
\hline No. of scaffolds & 8 & 21 \\
\hline Total length (bp) & $7,147,157$ & $8,384,694$ \\
\hline N50 & $1,183,318$ & 597,372 \\
\hline $\mathrm{GC}(\%)$ & 50.6 & 47.2 \\
\hline Average coverage & 278 & 38 \\
\hline Genome completeness $^{\mathrm{a}}$ & $92.8 \%(103 / 111)$ & $93.6 \%(104 / 111)$ \\
\hline Revised genome completeness ${ }^{\mathrm{a}}$ & $98.1 \%(103 / 105)$ & $99.0 \%(104 / 105)$ \\
\hline Genome contamination $^{\mathrm{a}}$ & $5.4 \%(6 / 111)$ & $6.3 \%(7 / 111)$ \\
\hline Revised genome contamination ${ }^{\mathrm{a}}$ & $0.0 \%(0 / 105)$ & $1.9 \%(2 / 105)$ \\
\hline Relative abundance in UASB metagenomes $(\%)^{\mathrm{b}}$ & 9.22 & 0.40 \\
\hline No. tRNA genes & 54 & 43 \\
\hline rRNA genes found in genome & $5 \mathrm{~S}, 16 \mathrm{~S}, 23 \mathrm{~S}$ & $5 \mathrm{~S}, 16 \mathrm{~S}, 23 \mathrm{~S}$ \\
\hline Inferred no. of rRNA operons ${ }^{c}$ & 3 & 2 \\
\hline No. CDS & 5989 & 7048 \\
\hline No. CRISPR array & 4 (125 repeats in total) & 5 (550 repeats in total) \\
\hline Coding density & $84.6 \%$ & $84.3 \%$ \\
\hline \multicolumn{3}{|l|}{ Putative glycoside hydrolases ${ }^{\mathrm{d}}$} \\
\hline Cellulase & $5(0.1 \%)$ & $14(0.2 \%)$ \\
\hline Amylase & $19(0.3 \%)$ & $8(0.1 \%)$ \\
\hline Debranching enzyme & $3(0.1 \%)$ & $2(0.1 \%)$ \\
\hline Amino-sugar-degrading enzyme & $35(0.6 \%)$ & $45(0.6 \%)$ \\
\hline Oligosaccharide-degrading enzyme & $43(0.7 \%)$ & $23(0.3 \%)$ \\
\hline \multicolumn{3}{|l|}{ Putative protease/peptidase $^{\mathrm{d}}$} \\
\hline Protease & $27(0.5 \%$ of total ORFs $)$ & $28(0.4 \%$ of total ORFs $)$ \\
\hline Peptidase & $60(1.0 \%)$ & $78(1.1 \%)$ \\
\hline \multicolumn{3}{|l|}{ Putative environmental signaling system genes } \\
\hline Transmembrane sensor ${ }^{\mathrm{e}}$ & $135(2.3 \%)$ & $114(1.6 \%)$ \\
\hline Response regulator containing CheY-like domain ${ }^{\mathrm{f}}$ & $131(2.2 \%)$ & $116(1.6 \%)$ \\
\hline Proposed class & Moduliflexia & Vecturatrichia \\
\hline Proposed order & Moduliflexales & Vecturatrichales \\
\hline Proposed family & Moduliflexaceae & Vecturatrichaceae \\
\hline
\end{tabular}

a. Genome completeness and contamination were estimated based on the presence/absence of a 111 single-copy gene set from Dupont et al. 2012. Revised genome completeness and contamination were calculated based on a revised total of 105 single-copy genes estimated to be present in the KSB3 genomes (Supplementary Table S2). Numbers in parentheses indicats detected number of genes per total number of each gene set. b. Relative genome abundance for each KSB3 genome was determined based on 16S rRNA gene profiling using shotgun metagenome data (Supplementary Sable c. Number of rRNA operons in the KSB3 genomes were inferred based on relative coverage profiles of KSB3 16S rRNA genes and the genome averages (Supplementary Fig. S2).

d. Counts ( $\%$ of total ORFs)

e. Predicted number of transmembrane sensors based on the possession of a sensor domain (Galperin 2004) and $>1$ transmembrane segments (Supplementary Table S8).

f. Number of all two-domain response regulators containing CheY-like domains estimated from PSI-BLAST searches of domain-specific profiles against the protein set described in Galperin 2004 (Supplementary Table S8). 